Ann. Génét. Sél. anim., I973, 5 (3), 299-3I2.

\title{
LES MUTANTS GÉNIQUES A EFFETS VISIBLES DANS LES RACES BOVINES D'ITALIE
}

\author{
G. SUCCI et J.-J. LAUVERGNE* \\ Istituto di Zootecnia Generale, Facoltà di Agraria, \\ Via Celoria 2, 20133 Milano (Italie) \\ * Laboratoire de Génétique factorielle, \\ Centre national de Recherches zootechniques, I. N. R. A., \\ 78350 Jouy en Josas (France)
}

RÉSUMÉ

Les principaux travaux consacrés aux mutations génétiques à effets visibles chez les bovins italiens concernent le culard en race Piémontaise et le nanisme achondroplasique en race Romagnole. Par le passé, des études ont également porté sur une hydrocéphalie en race Marchienne, une bride pénienne et une persistance de l'hymen en race Chianine et une anomalie costale en race Pisanne. Actuellement, des travaux sont en cours sur un type d'albinisme en race Brune des Alpes. Le comportement des gènes et leur fréquence, de même que les éventuels équilibres des fréquences géniques, sont encore imparfaitement précisés dans la plupart des cas. Les standards colorés, souvent identiques pour plusieurs races, sont expliqués en terme de mutants aux loci Agouti (A), d'albinisme vrai (C), de dilution (D) et des trois loci de panachure blanche (Bl, Blason, $\mathrm{S}$, Panachure irrégulière et $\mathrm{Cs}$, Flancs colorés).

Une réflexion moderne sur la génétique mendélienne des bovins domestiques fait apparaître deux grandes catégories phénotypiques de facteurs : les gènes à effets visibles et les gènes seulement décelables par des tests biochimiques et sérologiques.

Cette classification, qui peut paraître arbitraire, ne l'est que partiellement, car ces deux catégories se distinguent aussi par d'autres caractéristiques importantes, au moins sur le plan pratique.

Ainsi, les gènes à effets visibles ont, en général, des actions bénéfiques ou néfastes plus marquées que les gènes à effets invisibles et l'éleveur peut modifier, dans une certaine mesure, leurs coefficients de sélection génotypiques, influençant ainsi la fréquence à l'équilibre. Ces effets plus importants peuvent conduire à des applications pratiques intéressantes, mais sont, la plupart du temps, la cause d'anomalies.

Par le passé, l'étude des gènes à effets visibles a été faite sans grand ordre. Or, 
les races bovines modernes constituant des sortes d'isolats, il apparaît de plus en plus nécessaire de faire des études race par race. C'est ce que nous avons tenté de faire pour les bovins suisses dans un récent article (LAUVERGNE et WINZENRIED, I972). Le présent travail est consacré, quant à lui, aux races bovines italiennes.

\section{I. - MATÉRIEL E'T MÉTHODES}

\section{A. - Les sources}

Les auteurs italiens (Meregalli, 1956; Ghelardoni, I959; Pilla, I963; Galizzi Vecchiotti, I972j qui se sont penchés sur le problème des anomalies héréditaires des bovins l'ont fait dans une optique très générale d'éradication des récessifs indésirables, sans s'intéresser spécialement aux populations italiennes.

Il en est de même pour la coloration du manteau ou pour certains caractères comme l'hypotrychose : les articles de CenNi et Colombani (I969) et de Lucifero (I954) comportent les mêmes caractéristiques de cosmopolitisme que les articles évoqués ci-dessus.

Par contre, certaines monographies consacrées par Bonadonna (I959) et Calisti (I956, 1959, I965) à l'infécondité, et par RAIMONDI (1962) au culard Piémontais, rentrent beaucoup plus dans notre propos. Il a fallu les compléter par un dépouillement systématique d'Animal Breeding Abstracts. L'un d'entre nous (LAUvergne, 1967) a fait ce travail une première fois. Nous avons remis à jour sa liste pour faire le présent travail.

\section{B. - Mode d'exposé}

Parmi les gènes à effets visibles, il en est certains qui, comme les mutants de coloration, ont été fixés et font maintenant partie du standard des races. Toutefois, les combinaisons phénotypiques qu'ils déterminent sont beaucoup moins nombreuses que les races elles-mêmes, telles que nous les ont léguées les zootechniciens du siècle dernier. Aussi est-il plus commode pour cette catégorie de mutants de faire l'étude génotype par génotype.

Dans le chapitre suivant, consacré aux mutants en ségrégation qui sont responsables de variations anatomiques marquées, l'exposé est fait race par race.

\section{II. - LES MUTANTS RESPONSABLES DU STANDARD COLORÉ}

L'interprétation que nous donnons est basée sur les conceptions exposées par LAUVERGNE (I966).

Nombre de races bovines italiennes autochtones, dont certaines sont en voie de diminution, se caractérisent par une coloration blanche du pelage avec des plages grises (alliance de noir et de blanc) et des extrémités mélanisées; on les appelle parfois races podoliques. Ce sont, en se limitant aux races énumérées par BonadonNa (I959), la race Romagnole (Romagnola), la Chianine (Chianina), 1a Marchienne (Marchigiana), de la Maremme (Maremmana), la Pouillaise (Pugliese o Podolica Italiana), la Modénaise (Modenese o Bianca della Val Padana), la Piémontaise (Piemontese), la Grise Alpine (Grigia Alpina), la Grise de l'Adige (Grigia di Val d'Adige) et la Garfanienne (Garfagnina).

Pour ces races, l'interprétation génique déjà proposée pour la race Grise des steppes de Hongrie et pour la race Gasconne de France (LAUVERGNE, I966) nous semble encore valable. Les animaux porteraient le mutant $A^{y}$ (fauve à extrémité 
noire au locus Agouti), ainsi qu'un autre mutant, probablement au locus d'albinisme, homologue de 1'allèle " chinchilla " des rongeurs $\left(\mathrm{C}^{c h}\right)$ récessif et responsable de la disparition de la mélanine rouge (ou phœomélanine) des extrémités des poils, ne laissant subsister que la mélanine noire (ou eumélanine) de la base.

Certaines autres races, la Pisanne (Mucco Pisano), pourraient porter, au locus Agouti, l'allèle $\mathrm{A}^{w}$, le plus proche du type sauvage, comme la race Brune des Alpes (Bruna Alpina) venue de Suisse, qui, actuellement, est la deuxième race quant à l'effectif. Dans le génotype $\left(\mathrm{A}^{w}\right)$, les poils du manteau présentent des bandes qui font alterner la phœo- et l'eumélanine, le ventre et l'intérieur des membres restant clairs.

Chez d'autres races, on trouve le fauve à extrémités noires : $\mathrm{A}^{y} \mathrm{~A}^{y}$ au locus Agouti avec l'allèle normal au locus C d'albinisme : race Modicanienne (Modicana), Rendénienne (Rendena), Sarde (Sarda), et Tarine (Tarina) venue de Savoie.

On trouve également des phénotypes rouges ou noirs, généralement panachés.

\section{TABLEAU I}

Les formules géniques des colorations

du manteau des races bovines italiennes

\begin{tabular}{|c|c|c|c|c|c|c|}
\hline \multirow{2}{*}{ Races } & \multicolumn{6}{|c|}{ Loci } \\
\hline & A & $\mathrm{c}$ & $\mathrm{D}$ & $\mathrm{Bl}$ & s & Cs \\
\hline $\begin{array}{c}\text { Romagnole } \\
\text { Chianine } \\
\text { Marchienne } \\
\text { De la Maremme } \\
\text { Pouillaise, Modénaise } \\
\text { Piémontaise } \\
\text { Grise alpine } \\
\text { Grise de l'Adige } \\
\text { Garfanienne }\end{array}$ & $\mathrm{A}^{y} \mathrm{~A}^{y}$ & $\mathrm{C}^{c h} \mathrm{C}^{\mathrm{ch}}$ & ++ & $+t$ & ++ & ++ \\
\hline $\begin{array}{c}\text { Pisanne } \\
\text { Brune des Alpes }\end{array}$ & $\mathrm{A}^{w} \mathrm{~A}^{w}$ & ++ & ++ & ++ & ++ & ++ \\
\hline $\begin{array}{c}\text { Modicanienne } \\
\text { Rendenienne } \\
\text { Sarde, Tarine }\end{array}$ & $\mathrm{A}^{y} \mathrm{~A}^{y}$ & ++ & ++ & ++ & ++ & ++ \\
\hline $\begin{array}{c}\text { Frisonne Italienne } \\
\text { Pie Noire Valdôtaine } \\
\text { Burlinaise }\end{array}$ & $\mathrm{A}^{8} \mathrm{~A}^{8}$ & ++ & ++ & ++ & $\mathrm{S}^{p} \mathrm{~S}^{p}$ & ++ \\
\hline Pie Rouge Valdôtaine & $\mathrm{A}^{r} \mathrm{~A}^{r}$ & ++ & ++ & $\mathrm{B}^{1} \mathrm{~B}^{1}$ & $\mathrm{~S}^{p} \mathrm{~S}^{p}$ & ++ \\
\hline Pie Rouge du Frioule & $\mathrm{A}^{r} \mathrm{~A}^{r}$ & ++ & $\mathrm{D}^{f} \mathrm{D}^{f}$ & $\mathrm{~B}^{1} \mathrm{~B}^{\mathrm{I}}$ & $\mathrm{S}^{p} \mathrm{~S}^{p}$ & ++ \\
\hline $\begin{array}{c}\text { Reggianienne } \\
\text { Tortonaise Blonde }\end{array}$ & $\mathrm{A}^{r} \mathrm{~A}^{r}$ & ++ & $\mathrm{D}^{f} \mathrm{D}^{f}$ & ++ & ++ & ++ \\
\hline Pinzgau & $\mathrm{A}^{r} \mathrm{~A}^{r}$ & $\dot{+}+$ & ++ & ++ & ++ & $\mathrm{Cs}^{t} \mathrm{Cs}^{t}$ \\
\hline
\end{tabular}


Nous pensons que ces couleurs sont dues à deux allèles au locus Agouti : $\left(\mathrm{A}^{s}\right.$ pour le noir, $\mathrm{A}^{r}$ pour le rouge) et non à l'intervention d'hypothétiques mutants du locus $\mathrm{E}$ d'Extension, assez difficile à cerner, même chez les rongeurs, au contraire de ce que nous avons précédemment avancé (LAUVERGNE, I966). Ainsi, la Pie Noire Valdôtaine (Valdostana Pezzata Nera), la Burlinaise (Burlina), présenteraient le même génotype que la Frisonne (Frisona Italiana), d'importation récente, mais devenue la première race d'Italie : $\mathrm{A}^{s} \mathrm{~A}^{s} \mathrm{~S}^{p} \mathrm{~S}^{p}$ ( $\mathrm{S}^{p}$ étant le mutant récessif de panachure irrégulière).

Parmi les races dont le fond du manteau est rouge, on distingue des nuances plus claires qui seraient dues à un gène de dilution (que nous avons appelé $\mathrm{D}^{f}$ ) et des panachures. On trouverait, ainsi, la race Valdôtaine Pie Rouge (Valdostana Pezzata Rossa) avec la formule $\mathrm{A}^{r} \mathrm{~A}^{r} \mathrm{~S}^{\mathrm{P}} \mathrm{S}^{p} \mathrm{~B}^{l} \mathrm{~B}^{l}$, les panachures blanches étant du même type (corps à panachure irrégulière, tête blanche) que celles de la Pie Rouge du Frioule (Pezzata Rossa Friulana) qui forterait, en outre, le mutant pour la dilution $\mathrm{D}^{f}$, comme la Simmental. Dans cette perspective, les races Reggianienne (Reggiana) seraient $\mathrm{A}^{r} \mathrm{~A}^{r}$ et la Tortonaise Blonde (Bionda Tortonese) serait de formule $\mathrm{A}^{r} \mathrm{~A}^{r} \mathrm{D}^{f} \mathrm{D}^{f}$. La race Pinzgau (Pezzata Rossa Norica-Mölltal), originaire d'Autriche, porterait le gène de panachure blanche dit "flancs colorés" $\mathrm{C}^{\text {st }}$ et serait de formule $\mathrm{A}^{r} \mathrm{~A}^{r} \mathrm{C}^{s t} \mathrm{C}^{s t}$.

Le résumé de cette interprétation de la génétique de la couleur est donné dans le tableau I.

\section{III. - LES MUTANTS EN SÉGRÉGATION}

La présentation race par race va des races les plus nombreuses aux moins nombreuses. Les renseignements statistiques sur les races proviennent d'une enquête de I97I (XXVIII Assemblea A.I.A.).

\section{A. - Race Frisonne Italienne (Frisona Italiana)}

Depuis I963, c'est la première race italienne essentiellement à vocation laitière. Actuellement, elle groupe près de 3 millions d'individus. Les souches proviennent de Hollande, des États-Unis et du Canada.

Facteur rouge : un certain nombre de veaux pie rouge naissent. Jusqu'à présent, aucun travail n'a été fait pour estimer la fréquence de ce gène, qui est probablement le gène $\mathrm{A}^{r}$, précédemment retenu au génotype des races rouges, récessif par rapport au gène noir $\mathrm{A}^{s}$ et qui est bien connu des éleveurs dans les deux rameaux frisons d'Europe et d'Amérique du Nord.

\section{B. - Race Brune des Alpes (Bruna delle Alpi)}

Principalement à vocation laitière, elle comprenait I7 p. Ioo du cheptel en I970, occupant la deuxième place. En repli, devant la race Frisonne, elle est répanđue dans toute l'Italie, mais surtout dans les Vallées des Alpes.

Albinisme : décrit pour la première fois par ZaNOTris et al. (I967) dans la région du Trentin-Haut Adige, son étude se poursuit depuis cette date à l'Institut de Zoo- 
technie générale de la Faculté d'Agriculture de Milan (Rrzzotri et al., I969; BonaDONNA et al., I969 ; Succr, I973). Depuis 1972, les travaux sont couplés avec ceux qui se poursuivent sur le même sujet à l'Institut de Zootechnie de l'Université de Zurich, car il s'agit certainement du même mutant, beaucoup d'ancêtres des proposants italiens étant suisses et les caractéristiques anatomiques étant fort semblables.

C'est un mutant récessif vraisemblablement à pénétrance complète et expressivité assez constante. Le pelage tout blanc peut cependant, par exception, brunir avec l'âge. Le fond de l'œil est toujours rouge, l'iris est généralement vert clair dès la naissance. On a relevé detix anomalies concernant les trayons chez les femelles (I cas de 6 trayons; I cas de 2 trayons fonctionnels), mais, pour l'essentiel, les animaux albinos semblent avoir la même viabilité et les mêmes potentialités zootechniques que les sujets normaux (WEBER et LAUVERGNE, I964; WINZENRIED et LAUVERGNE, I968, I970).

La fréquence génique n'a pas encore pu être estimée en Italie.

Dès à présent, on a pu établir que ce type d'albinisme héréditaire différait génétiquement d'un autre albinisme apparu, quant à lui, en race Tachetée Rouge de Suisse (WEBER et al., I973).

\section{C. - Race Marchienne (Marchigiana)}

Avec 7,5 p. Ioo du cheptel, cette race grise, anciennement à double fin est orientée actuellement vers la production de viande.

Hydrocéphalie : de I943 à I952, Giannotri (I952) a décrit I 8 cas d'hydrocéphalie. Accompagnée parfois d'anomalie des organes reproducteurs, l'hydrocéphalie est souvent spectaculaire, avec un crâne énorme. Les animaux sont mort-nés ou vivent très peu de temps. L'examen des pedigrees laisse à penser qu'il s'agit d'un gène récessif autosomal, ce que semble confirmer PoLIDORI (I952). La fréquence du gène n'est pas estimée.

\section{D. - Race Piémontaise (Piemontese)}

Elle représente 7 p. Ioo du cheptel italien. Jadis cataloguée comme une race à triple fin (travail, lait, viande), sa spécialisation vers la production de viande s'accentue depuis quelques années, MaLETTO (I973).

Hypertrophie musculaire ou caractère culard : ce caractère est rapporté dans la race dès la fin du siècle dernier (Luciano, I903). Par ailleurs nous avons relevé la grande dispersion de ce mutant dans toutes les races européennes qui ont été, au siècle dernier, métissées avec la race anglaise Shorthorn (LAUVERGNe et al., I968).

Très tôt, les éleveurs piémontais se sont préoccupés d'acclimater et d'améliorer les performances bouchères des culards, multipliant leur nombre en dépit des anomalies qui accompagnent ce développement musculaire extrême (fig. I) (BrusaFERRO, I905 ; MARTINOLI, I905).

Dans les années vingt, on discutait encore de l'opportunité de développer ce caractère dans la race (Magliano, I923; Mascheroni, I923, I924; PaCr, I924), cependant que les avantages en boucherie, déjà bien décrits par MASCHERONI (I909), n'échappaient pas aux praticiens qui constituaient dès cette époque dans la région d'Alba (prov. de Coni, Piémont) (fig. 2) une variété entièrement culardisée, dite 


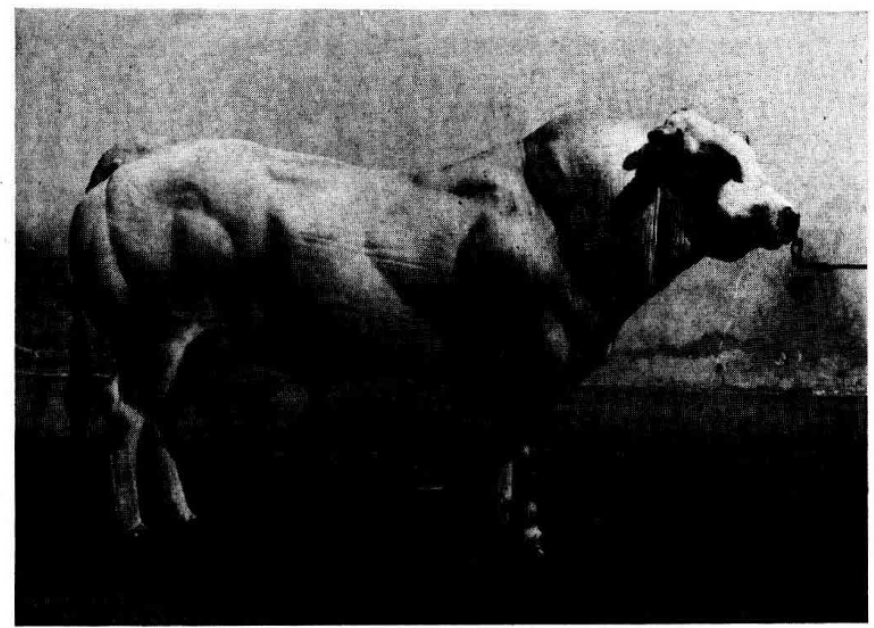

FIG. I. - Reproducteur Piémontais culard

On note l'extrême développement musculaire avec les sillons nettement marqués. Cette hypertrophie est encore plus accentuée par la réduction du tissu adipeux sous-cutané et la finesse de l'os. Le ventre est levretté.

(Photo : coll. Istituto Zooprofilattico Sperimentale di Torino)

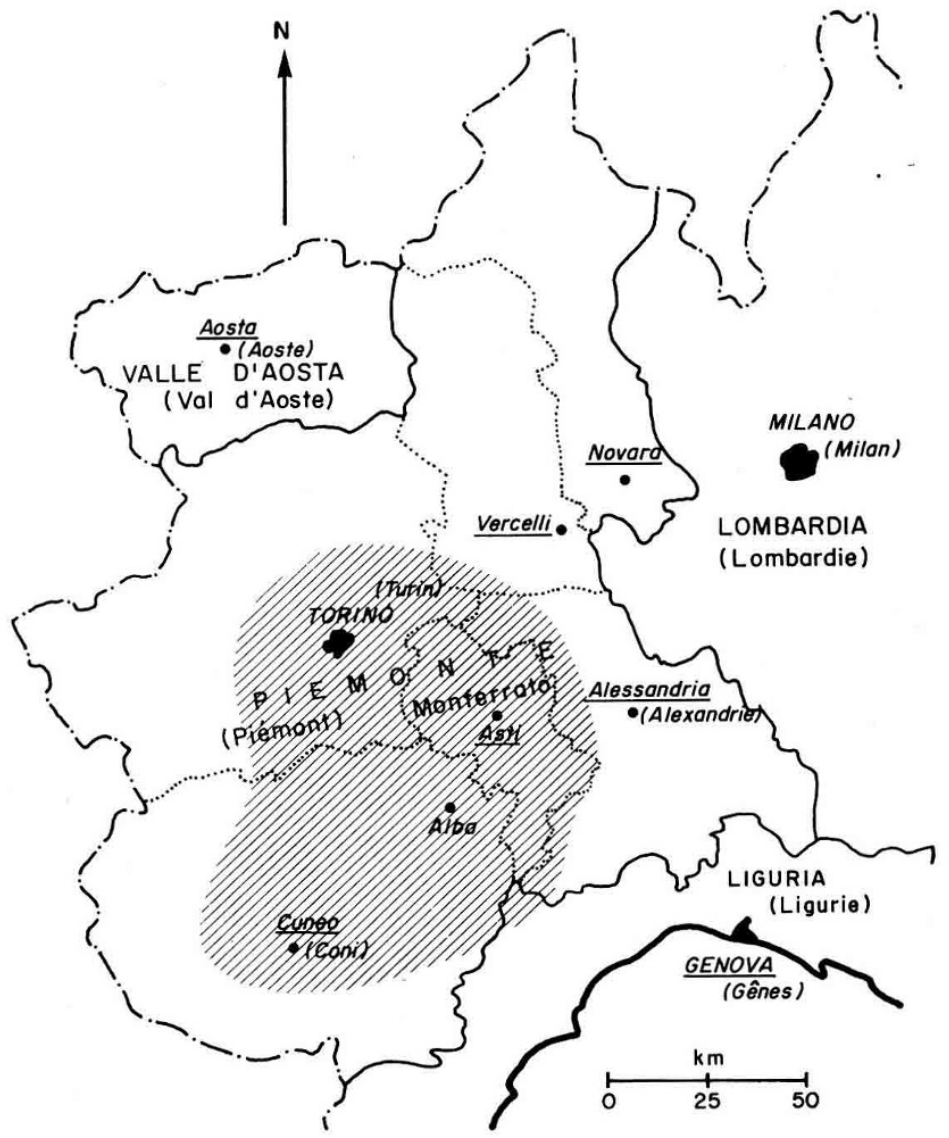

FIG. 2. - Extention de la race Piémontaise dans la région Piémont

En hachures, la principale zone de concentration dans les provinces d'Asti, de Coni (Cuneo) et Turin (Torino) surtout, avec un débordement sur Alexandrie (Alessandria). Les noms des chefs-lieux de pro* vince sont soulignés, entre parenthèses les noms francisés, quand ils existent. 
Albese, (VEZzani, I927; Magl,Iano, I933). La variété des appellations locales témoigne de la popularité de ce caractère : Della Coscia; GroppaDoppia; Groppa di Cavallo.

Dans les années trente, prennent place les premières études expérimentales: MALETTO (r934) et PACr (I935).

Par la suite, l'intérêt des chercheurs italiens, de Turin principalement, ne se démentira pas. La plupart des travaux, résumés une première fois par RAIMONDI en I957, puis en I962, sont consacrés à des expériences de rendement en viande et de croisement industriel à but pratique direct, avec souvent des répétitions et un brin d'emphase : Carbone (I940, I94I) ; RaImondi (I94I) ; Vezzani (I944) ; CaRBone (I947) ; ESMENARD et DASSAT (I949) ; PoRzIo (I952, I955) ; RAIMONDI (I955, I956, I960 $a$, et $b, \mathrm{Ig6} \mathrm{I}, \mathrm{I} 963, \mathrm{I} 964 a$ et $b, \mathrm{Ig} 65 a, b, c, d$, I970 $a, b$, I97I) ; UBERTALle (I955) ; CorTi (I956) ; Masoero (I960, I96I) ; ANONYME (I964) ; CaRbone et RAImondi (I965, I968, I970) ; DAssat et al. (I968) ; PARRUTi (I969) ; Casu (I970) ; Modena (I967, I970) ; Anonyme (I97 I $a$ et $b$ ) ; Auxilia et al. (I97I) ; Delfino (I97I) ; SUCCI et PEDRoN (I970) ; Bostrcco et BenatTi (I970).

Sur le plan génétique, une des meilleures études, bien qu'ancienne, reste celle de PACI (I935). Il ressort de celle-ci, comme des suivantes, qu'en race Piémontaise, le mutant qui, selon toute vraisemblance, détermine le caractère culard, est de type autosomal dominant à pénétrance incomplète et expressivité variable, l'homozygote étant généralement plus hypertrophié que 1'hétérozygote.

Les chercheurs italiens ont assez peu inventorié les défauts qui accompagnent le culard. Il est vrai que les qualités de la race Piémontaise semblent, dans une certaine mesure, atténuer les accidents les plus spectaculaires, au vêlage surtout. Toutefois, la fertilité et la production laitière des femelles culardes restent diminuées. On doit noter, cependant, les études de BorELII et CAGLIERo (I959) et Mol,INARI (I966) sur les césariennes, cependant que CENNI (I952) et MAGLIANo (I953) se sont préoccupés de la composition des os des culards et que VECchronI et TARTARINI (I960) ont amorcé des études sur le collagène du muscle.

Malgré toutes les études qui lui ont été consacrées, le caractère culard n'a pas révélé tous ses secrets : son appréciation reste encore imprécise et l'on n'a pas réussi à préciser l'origine du désordre physiologique qui entraîne la pléiotropie constatée.

Actuellement, en Italie, les recherches sur le culard Piémontais se poursuivent en plusieurs endroits. La principale orientation pratique est 1'utilisation de taureaux Piémontais culards en croisement industriel avec la Frisonne et la Brune des Alpes.

$\mathrm{Si}$, pour les femelles d'élevage Piémontaises, on essaie de garder l'ancien type, garant d'une carrière de reproduction moins heurtée, chez les veaux à l'engrais, par contre, la fréquence du caractère est très élevée (70 p. Ioo peut-être) à cause de l'emploi systématique de taureaux d'insémination très culards (cf. fig. I).

A l'étranger la race Piémontaise intéresse aussi les éleveurs et les chercheurs comme en témoignent certains articles français (ANONYME, I960, I964) et américains (ENSMINGER, I972).

\section{E. - Race Chianine (Chianina)}

Avec $4 \mathrm{p}$. Ioo du cheptel, c'est une race rattachable au groupe podolique à double fin (viande et travail), malgré les différentes hypothèses sur sa formation.

Bride pénienne : I5 jeunes taureaux de race Chianine observés à l'Institut de 
Pathologie spéciale et de Clinique vétérinaire de l'Université de Pérouse présentaient une bride pénienne interdisant mécaniquement le coït. Les pedigrees n'ont pas été analysés, mais on pense que l'anomalie est héréditaire (DozzA, I956). On ne dispose d'aucune donnée récente pour en estimer la fréquence dans la race.

Persistance de l'hymen : Oliva (I959, I96I) a observé un certain nombre de cas de cette anomalie chez des génisses de la variété Pérousienne. Il pense qu'il pourrait s'agir d'une anomalie héréditaire. Cela est confirmé par les anciennes observations d'AlunNo (I948) selon lesquelles cette tare aurait une fréquence de $2 \mathrm{p}$. Ioo en race Chianine et par l'observation plus récente de PANICHI (I965).

\section{F. - Race Romagnole (R̉omàgnola)}

Au début de I970, cette race, également de type podolique, groupait 2 p. Iod $\mathrm{du}$ cheptel bovin italien.

Achondroplasie : les premiers cas remontent au moins à I950 et, devant la régularité de leur apparition, le Ministère de l'Agriculture avait nommé en I956 une commission d'étude sous la présidence du professeur R. GivlianI, alors directeur de l'Institut de Zootechnie générale de l'Université de Florence. GERI et LUCIFERO (I964) ont donné la première relation détaillée de l'anomalie après que soient parues de brèves notes (BevilacQua, I956 ; Bendani, I957 ; Silvestri et CANTONI-Lughi, I959). Plus récemment, deux articles sont venus jeter un éclairage sur certains détails anatomiques : PEZzOLI et LEOPOLD (I966) et SEREN et al. (1972).

L'anomalie a une expressivité variable et les auteurs ont retenu 3 degrés

Io brachycéphalie et queue courte avec ou sans malformation des membres,

$2^{\circ}$ brachycéphalie, nanisme, queue courte et cassée, rétraction des tendons fléchisseurs et flexion des boulets,

$3^{\circ}$ hydrocéphalie, atrophie des mâchoires allant jusqu'à l'agnathie, atrichose, fissure palatine, torsion de la colonne vertébrale, amputation des membres, absence d'anus, début de colosomie (fig. $3 a$ et $b$ ).

Les mâles sont parfois stériles, les femelles le sont tout le temps. Les veaux sévèrement atteints meurent jeunes.

Même s'ils émettent des réserves, GERI et LUCIFERO (I964) considèrent finalement que l'anomalie est due, tout d'abord, à l'action d'un gène récessif autosomal. L'extension du caractère délétère dans la race résulterait alors, selon ces auteurs, de 1'action d'une certaine consanguinité alliée à une sélection en faveur de l'hétérozygote qui, par un effet dominant du gène serait plus " compact " et, partant, plus dans le type viande recherché des éleveurs.

L'éradication, courageusement commencée dès les années cinquante, n'est, semble-t-il, pas encore terminée.

Comme pour le Syndrome d'Arthrogrypose et de Palatoschisis que l'un d'entre nous étudie en race Charolaise, on peut se demander si, dans le cas de l'anomalie en race Romagnole, la pénétrance, implicitement supposée complète chez l'homozygote, l'est vraiment. En effet, les variations enregistrées dans l'expressivité laissent à penser qu'une partie des génotypes porteurs n'expriment pas la tare. Dans ces 

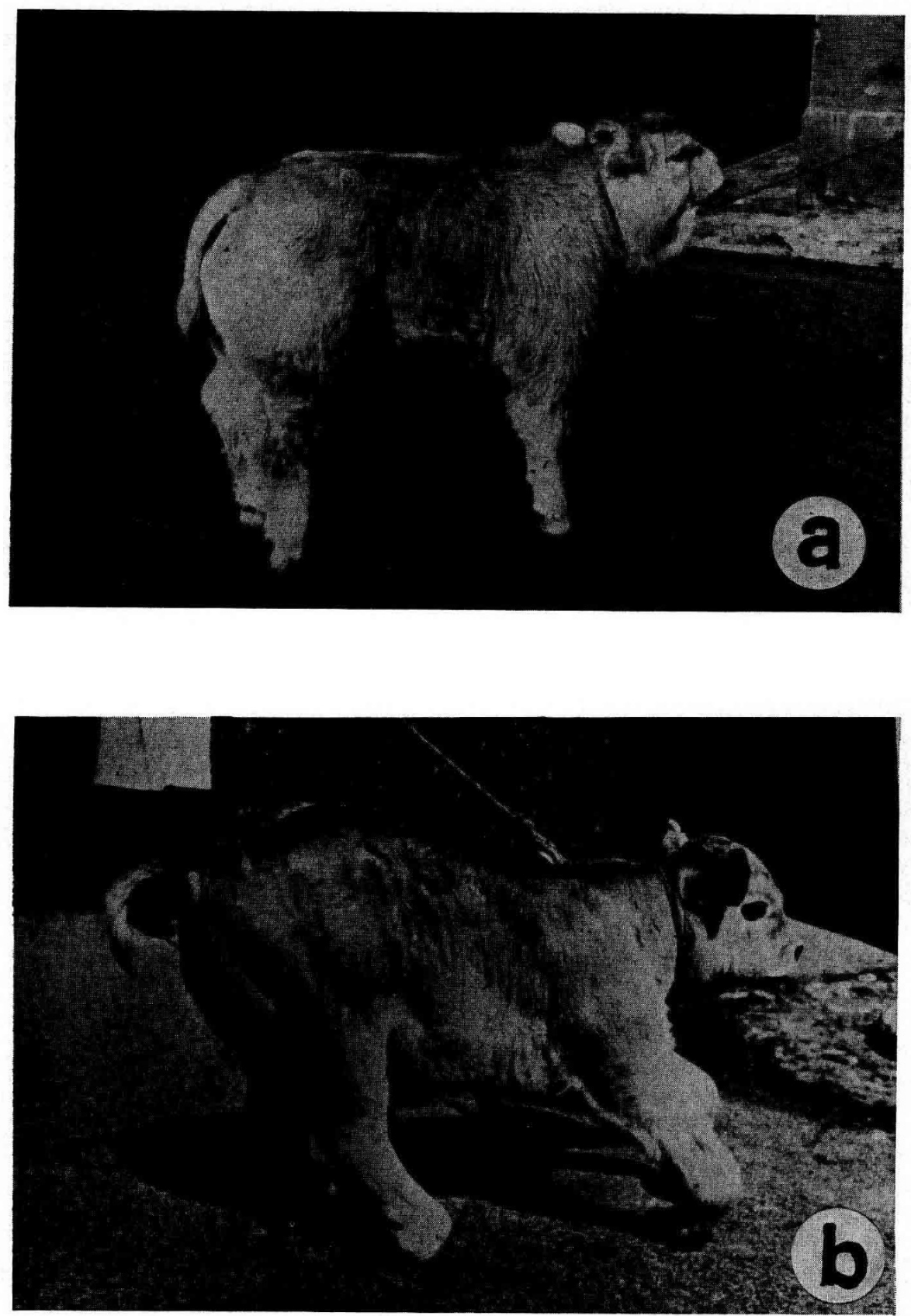

FIG. 3. - Le nanisme achondroplasique en race Romagnole

a) animal présentant le premier degré de l'anomalie : brachycéphalie, queue courte, membres subnormaux,

b) avec, en outre, la rétraction des tendons fléchisseurs et flexion des boulets, cet animal peut être classé dans le second degré de sévérité.

(Photos : coll. T. Bonadonna) 
conditions, certains phénomènes, difficilement explicables jusqu'alors, deviendraient plus compréhensibles, en particulier le contraste entre la fréquence relativement faible des tarés et la fréquence assez élevée des transmetteurs. De même, l'échec de l'éradication totale se comprendrait aisément, la détection des hétérozygotes se faisant assez tardivement, alors que l'animal, de toute façon (au moins en monte naturelle), a presque fini sa carrière de reproducteur.

On serait alors amené à se poser la question de savoir si les effets néfastes du gène ne sont pas compensés, et même au-delà, par des avantages invisibles (sur les caractères de reproduction en particulier) de l'hétérozygote (la femelle surtout). Une explication par un hétérosis invisible, mais économiquement avantageux, cadre mieux que l'explication par le choix des mâles hétérozygotes et la consanguinité pour rendre compte de la fréquence atteinte par le gène depuis sans doute assez longtemps. On peut, en effet, estimer que la population bovine Romagnole, surtout à une époque où l'insémination était peu répandue, devait présenter, pour le gène qui nous intéresse, un modèle quasi panmictique où l'influence du hasard due aux petits effectifs était bannie.

Il y a quelques années, RUGIATI et FEDRIGO ( 1967 ) ont décrit une double translocation chromosomique chez un taureau apparemment porteur de l'anomalie. Faute d'autres preuves, on peut toutefois penser qu'il s'agit d'une simple coïncidence et non pas de la cause habituelle de l'anomalie.

\section{G. - Race Pisanne (Mucco Pisano)}

Petit effectif (quelques centaines actuellement).

Anomalie costale : CenNi (I956), à l'abattoir de Pise, a observé quatre cas fort semblables d'une anomalie costale atteignant les cinq premières côtes. Le déterminisme héréditaire n'est pas précisé, pas plus que la fréquence de l'anomalie dans la race.

\section{CONCLUSION}

Le peu d'anomalies étudiées en Italie ne doit pas étonner. Jusqu'à une date récente, c'était une situation courante dans les pays voisins.

Ce qui peut surprendre, c'est que dans la principale race, la Frisonne, on n'en ait encore signalé aucune. Toutefois, la variété et le caractère récent des importations peuvent expliquer que les tares récessives tardent à apparaître.

Des deux études qui émergent du lot par leur importance, celle du culard, en race Piémontaise par l'ancienneté de sa mise en place, surprend favorablement. I a seconde. consacrée à l'achondroplasie en race Romagnole, peut sembler inachevée, mais le sérieux avec lequel les auteurs ont recueilli les données laisse à penser que 1es éléments d'une analyse plus complète sont déjà réunis. 


\section{SUMMARY}

\section{VISIBLE MUTANTS IN ITALIAN CATTLE BREEDS}

The main investigations of visible mutants in Italian cattle have involved double-muscled animals in the Piedmont breed and achondroplastic dwarfs in the Romagnola. Some work has also been done on hydrocephaly in the Marchigiana, cases of preputial constricture and of persistence of the hymen in the Chianina and of a rib abnormality in the Pisan breed. Work is in progress at the moment on albinism in the Brown Swiss. In the majority of cases the genetic behaviour of the mutants and their frequency, let alone their eventual equilibrium frequency, are very uncertain. Variants of colour pattern, often identical in several breeds, are explained in terms of mutations at the loci of Agouti (A), true Albinism (C), Dilution (D) and three loci affecting extension of white pigment (Bl, Blaze, S, Irregular Spotting and Cs, Colour-sided).

\section{RIASSUNTO}

\section{MUTANTI GENICI AD EFFETTO VISIBILE NELLE RAZZE BOVINE ITALIANE}

I principali lavori dedicati ai mutanti genici ad effetto visibile nelle razze bovine italiane concernono il carattere "culard " nella Piemontese ed il nanismo acondroplasico nella Romagnola. In passato, altri studi sono stati rivolti all'idrocefalia nella Marchigiana, alla persistenza dell'imene nella Chianina e ad anomalie costali nella Pisana. Attualmente sono in corso ricerche sull'albinismo nella razza Bruna Alpina. Il comportamento dei geni e la loro frequenza, come gli eventuali equilibri delle frequenze geniche, nella maggior parte dei casi, non sono ancora precisati chiaramente. Gli standards relativi alla colorazione del mantello, spesso comuni a più razze, vengono interpretati in termini di mutanti dei loci responsabili del colore Agouti (A), dell'Albinismo vero (C), della Diluizione (D) e dei tre loci responsabili della pezzatura bianca : $\mathrm{Bl}$ (Testa bianca), S (Pezzatura irregolare) e Cs (Fianchi colorati).

\section{RÉFÉRENCES BIBLIOGRAPHIQUES}

Alunno E., r948. Contributo allo studio statistico delle cause di infecondità non patologica nelle bovine di razza chianina. Zoot. Vet., $3(6), 23$.

Anonyme, 1960. Amélioration de la production de la viande dans la province de Piémont par l'utilisation de taureaux culards. Union nationale des Livres généalogiques, Paris, 60 p. ronéoté.

Anonyme, I964. Note sur les résultats du contrôle de descendance des taureaux Piémontais "della coscia ". effectué à la C. A. D. I. A. du Gers. Centre technique pour le contrôle de la Descendance, Paris, c. r. $n^{0} 58$.

Anonyme, I97 $a$. La razza bovina Piemontese " della coscia". La razza bovina Piemontese, $2(6)$.

Anonyme, r97 $\mathrm{r}$ b. Prove di progenie sulla razza bovina Piemontese. La razza bovina Piemontese, 2 (ro), I $69 \mathrm{p}$.

Antonelli A., r96I. Le mostruosità nella razza Romagnola, breve cronistoria ed osservazioni tecniche. Riv. Zoot., 34, $221-225$.

Auxilia M. T., Maletto S., Mussa P. P., Raimondi R., I97I. Dati sui seguenti parametri : incremento ponderale, indici di conversione degli alimenti, indici biometrici, resa alla macellazione, allo spolpo ecc., esame del taglio campione, caratteri chimici, bromatologici delle carni. La razza bovina Piemontese, 2 (10), 63-134.

Bendani N., 1957. Relazione alla $1^{\text {a }}$ riunione della commissione per l'indagine sulla comparsa di soggetti mostruosi o malformati nella razza bovina Romagnola. Cesena, 12 gennaio 1957.

BevilacQua G., r956. Relazione sulle malformazioni congenite in vitelli di razza Romagnola in provincia di Forli. Ispettorato provinciale dell'Agricoltura di Forli.

Bonadonna T., r959 a. Fattori genetici ed infecondità dei bovini. Progr. Veter., 14, 33 I-343.

Bonadonna T., r959 b. Le razze bovine. Edizioni " Progresso Zootecnico », Milano, ro37 p. 
Bonadonna T., Roy Choudhury P. N., Succi G., I969. Osservazioni su talune caratteristiche del materiale spermatico di un toro albino di razza Bruna delle Alpi. Boll. Soc. ital. Biol. Sperim., 46, 49-5 I.

Borelli G., Cagliero G., I959. L'opération césarienne chez les vaches dans la zone du Piémont (Italie) où on élève le veau "de la cuisse " ou à croupe de cheval ("Doppeliender »). Statistiques et considérations opératives au regard des techniques effectuées auprès des élevages. 16th Int. vet. Congr., Madrid, 2, 95 I-952.

Bosticco A., Benatti G., I97o. Possibilità offerte dall'incrocio di prima generazione alla produzione della carne bovina in Italia. Relazione al $3^{\circ}$ Symposium di Genetica Animale, Asti, I I maggio.

Brusaferro S., I905. I vitelli Piemontesi a groppa di cavallo alla prova di macellazione. Moderno Zooiatro, 25, I35-127, 228-233, 248-253.

Calisti V., I956. Fattori ereditari e congeniti dell'infertilità maschile. Proc. 2nd World Congr. Fert. Ster., Napoli, roI I-I038.

Calisti V., I959. Forme ereditarie di sterilità nei bovini. Atti Soc. ital. Sci. vet., 13, 53-136.

Calisti V., 1965. Le malformazioni genitali nei bovini. Atti. Soc. ital. patologia, 45-I26.

Carbone E., r940. Prove di ingrassamento comparative di vitelli Piemontese comuni, Piemontese a groppa di cavallo e meticci Charolais $\times$ Piemontese. Annali Sper. Agr. I9I-208.

Carbone E., I94I. La produzione del vitello grasso in Piemonte. Italia agric., 78, III-I30.

Carbone E., I947. I bovini Piemontesi a groppa di cavallo. $2^{\circ}$ Convegno agr. Alba. Ott. I946. Zootec. vet., 2, I67-168.

Carbone E., Raimondi R., I965. Saggio di controllo genetico di tori Piemontesi della coscia. Riv. Zootec., 38, I64-187.

Carbone E., Raimondi R., i968. Problemi di allevamento, alimentazione e produzione della razza bovina Piemontese. Riv. Zootec. A gric. Vet., 6, 85-98.

Carbone E., Raimond R., I97o. Prova di ingrassamento di vitelloni Piemontesi " della coscia " con impiego di urea in parziale sostituzione dell'azoto proteico. Annali Istituto Sper. Zootecnia, 3, 9 I-I04.

Casu S., I97o. L'incrocio tra tori di razza Charolaise, Piemontese, Limousine e bovini locali nella produzione della carne in Sardegna. Convegno su problemi degli allevamenti bovini ed ovini in Sardegna.

CENNi B., I952. Studi comparativi sulla composizione delle ossa dei bovini " a groppa di cavallo" e bovini comuni del Piemonte. Ann. Fac. Med. Vet., Pisa, 5, 36-6o.

Cenni B., 1956. Di una particolare malformazione in bovini di razza Pisana. Ann. Fac. Med. Vet., Pisa, 9, I56-160.

Cenni B., Colombani B., I969. L'eredità del colore del mantello nei bovini. Riv. Zootecnia, 42, 540, $622-672$.

Corti L., r956. Per il miglioramento della razza bovina Piemontese. Progresso vet., 11, 584-588.

Dassat P., Hodges J., Mason I. L., Vissac B., 1968. Miglioramento genetico della razza bovina Piemontese. Riv. Zootec., Agric. vet., 6, 149-I 59.

Delfino F., I97I. Il bovino piemontese della coscia nella produzione della carne. La razza bovina piemontese, 2, 17-23.

Dozza G., 1956. Impotentia coeundi da causa meccanica congenita in tori di razza Chianina. Nuova Vet., 32, 252-255.

Ensminger M. E., I972. Double muscled cattle. Good or bad? Cattlemen, 35 (6), I4-15, 44-45.

Esmenard G., Dassat P., r949. La selezione del bestiame bovino in Piemonte. Congr. agr. naz., Torino, 14-17, ott. 1948, 52 p.

Galizzi Vecohiotid G. A., I972. Su l'individuazione del riproduttore portatore di caratteri indesiderabili. Riv. Zootecn., 45, $201-2 \mathrm{I} 6$.

GERI G., LUCIFERO M., I964. Indagini sulle anomalie di tipo acondroplasico comparse nella razza bovina Romagnola $e$ sul lavoro di risanamento genetico. Centro di studio per la genetica animale del C. N. R. Istituto di Zootecnia Generale dell'Università di Firenze, r 57 p.

Gherardon E., I959. Elenco dei principali caratteri letali e sub-letali osservati in animali agricoli. Ann. Fac. Med. vet., Pisa, 12, 220-238.

Grannotrı D., I952. Casi di iđrocefalo congenito in vitelli. Memor. Soc. tosc. sci. nat. B., 59, séance du 25 juin 1952 .

Lauvergne J. J., I966. Génétique de la couleur du pelage des bovins domestiques (Bos taurus, LinNé). Bibliographia Genetica, 20, I-68.

LAUVERGNE J. J., I967. Liste des travaux concernant les anomalies congénitales, héréditaires ou non des bovins dans le monde depuis 1900 (édition provisoire). Commission de génétique. Sessions des commissions d'études de la F. E. Z., Oslo, I4-16 août $1967,93 \mathrm{p}$.

Lauvergne J.-J., Boyazoglu J.-G., Hubert D., I968. Le phénomène culard chez les bovins : bibliographie annotée. Bull.tech. Dép. Génét. anim. (Inst. nat. Rech. agron., Fr.) n ${ }^{0} 2$.

LAUVERGNe J. J., Winzenkied H. U., r972. Les mutants géniques à effets visibles dans les races bovines de Suisse. Ann. Génét. Sél, anim., 4, 523-535.

Luciano E., r903. Vitelli a groppa doppia o di cavallo. G. R., Soc. Accad. vet. ital., 52, 793-806.

Lucifero M., r954. L'ipotricosi nei bovini. Riv. Zootec., 27, 8r-82.

Magliano A., I923. I bovini a groppa di cavallo. G. med. vet., 72, 533-536. 
Magliano A., r933. Indagini circa il comportamento genetico della cosidetta " Groppa di cav allo nei bovini di razza Piemontese. Clinica vet., Milano, 11, I24-I39.

Magliano A., I953. Études comparatives sur la composition des os de bovins à " cul de poulain " doppellender et ceux des bovins normaux du Piémont. $X V$ int. vet. Congr. Stockholm, 126.

Maletto F., 1934. Experimenti di incrocio industriase tra la razza bovina Charolaise e la razza Piemontese. Aratro, Alessandria, 11 (3I).

Maletro s., I973. Origine ed evoluzione formativa dei bovi di la razza bovina Piemontese. La Razza bovina Piemontese, $4(5-6) 7 \mathrm{I}-130$.

Martinoli G., r905. I bovini " doppellender ». Moderno Zooiatro, 25, 431-432.

Mascheroni E., Igog. I bovini a groppa di cavallo. Studi a rilievi fatti sull'allevamento di questi animali per la produzione della carne. Cronaca $\mathrm{Agr}$., 21-22.

Mascheroni E., r923. Si deve incoraggiare o prescrivere come pericoloso ai nostri allevamenti la produzione dei bovini della coscia a groppa di cavallo. Ye Congr. allev. razza bovina Piemontese, Mondovi.

Mascheroni E., r924 a. Horse-rumped cattie at the Piemontese breeder's congress. Mon. Bull. int. Inst. Agric., Roma, 2, 154-156.

Masoero P., ig6o. Ii contributo della razza bovina Piemontese alla soluzione del problema della carne. Convegno naz. per $i$ problemi della carne, Firenze, 448-450.

Masoero P., Ig6r. Caratteri ed elementi di stabilizzazione del vitello della coscia di razza Piemontese. Giornata Nazionali Veterinarie, Saluzzo, Sett.

Meregalli A., r956. I fattori letali e subletali identificati nei bovini. Riv. Zootec., 36, $289-290$.

Modena A., 1967. I bovini di razza Piemontese. Convegno per il miglioramento della razza bovina Piemontese, 43 p.

Modena A., I97o. Indagine sulla razza bovina Piemontese con particolare riguardo al tipo " della coscia ". Progr., vet., 25, 99-102.

Molinari G., I966. Osservazioni sul taglio cesareo nelle bovine di razza Piemontese con particolare riguardo alla fertilità nelle operate ed i caratteri morfo-genotipici dei vitetli cosidetti " della coscia ". Progr. vet., 21, 44I-446.

Oliva O., 1959. Anomalia congenita in giovenche Chianino-Perugine: persistenza dell'imene. Atti Soc. ital. Sci. vet., 13, 224-228.

Oliva O., Ig6I. Ulteriore osservazione di casi di persistenza dell'imene in manze Chianino-Perugine. Symp. Inter. Sterilità. Minerva Medica, Torino.

PACI C., r924. Relazione sui bovini a groppa di cavallo. Primo Congr. Zootec. Piemonte, Cuneo.

PACI C., I935. Indagini e controlli sulla formazione della sottorazza Albese. Riv. Zootec., 12, I49-I54, I59, 197-202, 207, 208, 240-250, 255.

Panichi G., I965. Malformazioni genitali in una manzetta Chianina riportabili alla " Maladie des génisses blanches." Ann. Fac. Med. Veter., Pisa, 18, 265-273.

Parruti B., I969. Prova di incrocio industriale del toro Piemontese a della coscia " con bovine di razza Marchigiana. L'informatore agrario, 25, 1227-1230.

Pezzoli G., Leopold A., I966. Il nanismo acondroplasico nella specie bovina. Quadro radiologico. La Nuova Veterinaria, 42, 3-19.

Pilla A. M., I963. I geni recessivi indesiderabili nelle popolazioni degli animali domestici. Ann. Sper. Agr., 17, 413-432.

Polidori F., 1952. Malformazioni congenite in vitelli. Atti Soc. tosc. Sci. Nat., 59, série B., séance du 15 déc. 1952 .

Porzio G., I952. I bovini Piemontesi della cosidetta sottorazza Albese. L'allevatore, 8, 33-34.

Porzio G., 1955. Aspetti tecnici della produzione della carne in Piemonte. 32e Convegno naz. Soc. ital. Progr. Zootec., Chivasso, 13 dicembre, $2 \mathrm{I}-3 \mathrm{I}$.

RaImondi R., r94I. Studio sulla resa alla macellazione di vitelli Piemontesi di tipo, di sesso e di età diversi. Nuovi Annali Agric., 21, 33 p.

Rarmondi R., I955. Nuovi elementi relativi alla produzione dei bovini Piemontesi cosidetti " della coscia ". 32e Convegno naz. Soc. ital. Progr. Zootec. Chivasso 13 dicembre, 3 I-35.

Raimondi R., I956. La razza bovina Piemoniese e le sue attuali possibilità di miglioramento. Riv. Zootec., 29, 324-329.

Raimondr R., r957. Studio sui bovini Piemontesi " a groppa doppia ". Annali Accad. Agric., Torino, 99, $60 \mathrm{p}$.

Raimondi R., I960 a. Prospettive di impiego del toro Piemontese " della coscia " per l'incrocio industriate con le razze da latte. Annali Accad. A gric., Torino, 102.

RaImondi R., I96o $b$. I bovini Piemontesi " della coscia "in rapporto al problema della carne. Convegno naz. per $i$ problemi della carne, Firenze, 502-52 I.

RaImondr R., I96r. Esperienza comparativa di ingrassamento e di macellazione tra vitelli Piemontes $i$ a "groppa doppia " e comuni. Annali Sper. Agr., 15, 150-151.

RaImondi R., 1962. I bovini Piemontesi a groppa doppia. G. B. Paravia, Torino, 92 p.

RaImondi R., ig63. Risultati di una prova di allevamento di bovine Piemontesi del tipo "della coscia ". Annali Sper. Agr., 17. 471-489. 
Raimondi R., r964 a. Risultati di una prova di incrocio industriale fra un toro Piemontese a " groppa doppia " e vacche di razza Valdostana Pezzata Rossa. Riv. Zootec., 37, II7-I42.

Raimond ., I964 b. Attuale evoluzione tecnica e produttiva dell'allevamento bovino in Piemonte. Riv. Zootec., 37, 589-600.

Raimondi R., r965a. Résultats des essais de croisements entre des taureaux Piémontais de boucherie et des vaches de races laitières. Wld Rev. Anim. Prod., I no spec., 89-9r.

Raimondi R., I965 b. Prova comparativa di ingrassamento fra vitelloni meticci Piemontese della Coscia $\times$ Frisona e vitelloni di razza Frisona. Riv. Zootec., 38, I1 I-I27.

Raimondi R., I965 c. Influenza del peso di macellazione sulla produzione economica di vitelli $P$ iemontesi all'ingrasso. Riv. Zootec., 38, 443-448.

Raimondi R., I965 d. Attuale situazione della razza bovina Piemontese. Riv. Zootec., 38, 563-572.

Rarmondr R., r97o a. Attuale situazione della razza bovina Piemontese. L'informatore Agrario, 26, (I8), I 533-I 535 .

RaImondi R., r97o $b$. Problemi genetici nelle prospettive di miglioramento della razza bovina Piemontese. 3e simp. naz. Genet. anim., Asti, 11 maggio.

Raimondi R., r97I. Studio sulle attitudini riproduttive e produttive delle meticce di $\mathrm{I}^{\circ}$ incrocio $P$ iemontese "della coscia" $\times$ Frisona. La razza bovina Piemontese, 2 (3), 26-27.

Rizzotti M., Aureli G., Zanotri L., Straini Pannelli R., Succi G., ig69. Ricerche morfologiche e istochimiche sulla pelle e biofisiche sui peli di bovini albini. Riv. Istoch. Norm. pat., 15, 91-I Io.

Rugiati S., Fedrigo M., 1967. Alterazione cromosomica riscontrata in un toro acondroplasico di razza Romagnola. Ateneo Parmese Acta bio-medica. 38 (5), 5 p.

Seren E., Matteuzzi A., Falaschini A. F., I972. Funzionalità tiroidea di bovini Romagnoli nani acondroplasici. La Nuova Veterinaria, 48, I Io-I 9.

Silvestri A., Cantoni lughi G., i959. Consanguineità e teratologia. Progr. vet., 16 (19), $725-728$.

Succi G., 1973. Contribution à l'étude de l'albinisme en race Brune des Alpes. Ann. Génét. Sél. anim., 5 , I43-I 45 .

Succi G., Pedron G., I970. Risultati di prime prove di incrocio tra bovine di razza Frisona pezzata nera e tori delle razze Piemontese "della coscia " e Charolais. Bull. Agricolt., 105 (23), I-2.

Ubertalle A., I955. Alcuni aspetti della produzione e del consumo della carne bovina in Piemonte. Annali Fac. Med. Vet., Torino, 5, III-II2.

Vecchioni R., TARTARIN A., r96o. Comportamento delle frazioni collagene nella ipertrofia muscolare. Boll. Soc. Ital. Biol. Sper., 26, I4I5-I4I7.

Vezzani V., 1927. La formazione della sottorazza albese in seno alla razza bovina Piemontese. Riv. Zootec., 4, 3I 3-2 I9, 259-266.

Vezzanr V., I944. Ricerche sperimentali sulla produzione della carne bovina in Piemonte. Istituto sperimentale zootecnico di Torino.

Weber W., Lauvergne J.-J., I964. Trois cas d'albinisme rencontrés en Suisse dans la race Brune des Alpes. Ann. Zootech., 13, I5I-I54.

Weber W., Lauvergne J.-J., Winzenried H. U., I973. Albinisme héréditaire en race Tachetée Rouge de Suisse. Schweiz. Archiv. Tierheilk., 115, I42-I44.

Winzenried H. U., Lauvergne J.-J., 1968. Albinism among Brown Swiss cattle. XII Int. Conf. Genet., 1, 280.

Winzenried H. U., Lauvergne J.-J., I97o. Spontanes Auftreten von Albinos in der schweizerischen Braunviehrasse. Schweiz. Arch. Tierheilk, 112, 58 I-587.

Zanotti L., Aureli G., Rizzotti M., Succi G., I967. Osservazioni su peli di bovini albini mediante lo studio di spettri EPR. Kiv. Istoch. Norm. Pat., 13, 45I-46o. 